\title{
Dissemination of responsible service of alcohol initiatives to rugby league clubs
}

\section{ABSTRACT}

OBJECTIVES: To determine the acceptability of intervention strategies that increase the responsible service of alcohol by nonmetropolitan rugby league clubs. METHOD: Rugby league clubs were provided an information kit and advice by local public health workers, police and a lead agency regarding their responsible service of alcohol practices. Rugby League clubs and public health workers completed an acceptability survey at the conclusion of the study.

RESULTS: Data were collected from 160 contactable clubs ( $100 \%$ consent) and 12 of 14 participating public health workers. Almost all clubs reported of contact with the lead agency, public health workers and police to be acceptable. Fifty-nine percent of clubs reported contact with public health workers to be useful. One-third of the public health workers considered that they were not the most suitable professional group to be involved in delivering the intervention.

ConcLusions: In spite of a suggested culture of harmful alcohol consumption among rugby league participants and spectators, non-metropolitan rugby league clubs appear receptive to public health strategies that increase their responsible service of alcohol.

IMPLICATIONS: The ability of the public health sector to meet this opportunity appears limited, and may require additional strategies to increase the capacity of public health workers to develop/deliver inter-sectoral interventions in this setting.

\author{
Matthew Warner-Smith \\ Hunter Centre for Health Advancement, Newcastle, New South Wales
}

\section{John Wiggers}

Hunter Centre for Health Advancement, Newcastle, New South Wales and Faculty of Medicine and Health Sciences, University of Newcastle, New South Wales

\section{Robyn Considine, Jenny Knight}

Hunter Centre for Health Advancement, Newcastle, New South Wales

\section{Introduction}

A lcohol consumption is a significant contributor to mortality, morbidity and reduced quality of life in Australia, particularly among males, people aged 18 and 34 years, those residing in non-metropolitan areas and those of low socio-economic status. ${ }^{2}$

Enhancing the responsible service of alcohol by licensed premises has been suggested to be effective in reducing service to intoxicated patrons, ${ }^{3}$ patron blood alcohol levels ${ }^{4}$ and alcohol-related traffic accidents. Strong community and industry support exists for the responsible service of alcohol in licensed premises. ${ }^{67}$ Although inclusion of responsible service initiatives in licensing laws is an increasingly common approach to reducing harm, ${ }^{8}$ the introduction of legislation alone is unlikely to be effective in the absence of adequate enforcement and strategies that enhance the knowledge and skills of licensees. ${ }^{6.9}$

Sporting organisations represent an appropriate setting for reducing alcohol-related harm as 9.3 million Australians are estimated to attend or participate in sports events annually. Those who attend or participate in sports events are at considerably greater risk of alcohol-related harm. ${ }^{10-13}$

Despite the potential for sporting groups to contribute to reducing alcohol-related harm, this potential has yet to be realised. ${ }^{10} \mathrm{~A}$ first step towards achieving this objective is to develop acceptable strategies that facilitate the adoption of responsible service initiatives by such groups. ${ }^{14}$ Given the relative absence of reported data in this area, ${ }^{10}$ this study sought to determine the acceptability of strategies designed to increase the responsible service of alcohol by rugby league clubs, as reported by rugby league clubs and participating public health workers.

\section{Methods}

SAMPLE AND SETTING

Rugby league clubs in New South Wales who participated in the 1997 New South Wales Country Rugby League competition were eligible to participate in the study.

Each of 11 non-metropolitan health areas or districts in New South Wales was eligible to participate in the study if they reported that they had the capacity to deliver the intervention to clubs in their area.

\section{INTERVENTION}

The intervention consisted of the following 4 components:

- A Responsible and Safe Drinking Information Kit was developed by the Hunter Centre for Health Advancement (lead agency), NSW Police Service, the New South Wales Department of Racing and Gaming and 2 rugby league clubs.
Submitted: July 1999

Revision requested: November 1999 Accepted: January 2000

\section{Correspondence to:}

Dr John Wiggers, The Hunter Centre for Health Advancement,

Locked Bag 10, Wallsend NSW 2287

Fax: (02) 4924 6215. Email; jwiggers@doh.health.nsw.gov.au 
- Local public health workers trained in the delivery of the intervention were asked to mail the information kit to each rugby league club in their area, and to contact each club on 2 subsequent occasions to offer on-going support.

- All police districts in non-metropolitan areas were mailed the information kit by the lead agency and asked to audit the alcohol service practices of rugby league clubs in their area.

- Clubs were telephoned by the lead agency after 3 months to assess the adoption of the recommended practices. Feedback and advice were offered to those clubs that reported the non-adoption of practices.

\section{Measures}

INTERVENTION ACCEPTABILITY AS REPORTED BY RUGBY LEAGUE CLUBS

Six months following the distribution of the information kits, all eligible clubs were asked to participate in a telephone survey (maximum of 5 call attempts). Clubs were asked if they sold or supplied alcohol, and whether or not they had been contacted by a local public health worker or police officer regarding responsible alcohol service practices in the previous 6 months. Clubs were asked to indicate the acceptability of the information kit, the contact by the lead agency and the contact by local public health workers and police. Where applicable, clubs were asked to report the usefulness of the intervention components (six 4point Likert scale items - very, moderately, slightly, not at all useful).

\section{TABLE 1: PROPORTION OF CLUBS REPORTIMG THE INTERVENTION} COMPONENTS TO BE USEFUL

\begin{tabular}{lcc}
\hline & \multicolumn{3}{c}{ Very/moderately useful } \\
& $\mathrm{n}$ & $\%$ \\
\hline Public health worker contact useful? & 96 & 59 \\
\hline Police contact useful? & 82 & 96 \\
\hline Kit useful? & 135 & 88 \\
\hline Kit easy to use? & 133 & 96 \\
\hline Information in kit credible? & 134 & 98 \\
\hline Kit easy to understand? & 134 & 99 \\
\hline
\end{tabular}

INTERVENTION ACCEPTABILITY AS REPORTED BY PUBLIC HEALTH WORKERS

Following the completion of the project, public health workers completed a questionnaire to determine the acceptability (six binary items - yes/no), and usefulness of the intervention components (eight 4-point Likert scale items - very, quite, slightly, not at all useful), aggregated to useful (very, quite).

\section{Results}

\section{SAMPLE}

\section{Rugby League Clubs}

Two hundred and seven rugby league clubs were eligible to participate in the study. Seven clubs were excluded (5 in a health area unable to deliver the intervention and 2 that participated in the development of the intervention). Of the remaining 200 clubs, $160(80 \%)$ were able to be contacted by telephone after 5 call attempts, all of whom consented to participate in the survey. Clubs located in major urban centres (Newcastle, Wollongong and NSW Central Coast) were more likely to be contacted $(97 \%$ of 26$)$ than those in the remaining rural areas ( $84 \%$ of 112$)$.

\section{Public Health Workers}

Public health workers employed by the lead agency were excluded from the study, as were workers from one health district due to its reported inability to deliver the intervention.

Fourteen public health workers participated in the study from the 9 remaining health areas/districts ( $7 \mathrm{drug}$ and alcohol officers, 6 health promotion, 1 alcohol and road safety officers). Two drug and alcohol officers were unable to be contacted for the post-study data collection.

\section{INTERVENTION ACCEPTABILITY AS REPORTED BY RUGBY LEAGUE CLUBS}

Results are reported for the 135 clubs $(84 \%)$ that sold or provided alcohol to players or supporters.

Seventy-one percent of rugby league clubs reported being contacted by a public health worker and $61 \%$ reported being contacted by local police. Almost all clubs reported that contact by the lead agency (93\%), a public health worker $(90 \%)$ and police $(99 \%)$ was acceptable. Reported usefulness of the contact is described in table 1. No differences were evident in the reported acceptability and usefulness of contact between clubs located in urban and rural areas.

INTERVENTION ACCEPTABILITY AS REPORTED BY PUBLIC HEALTH WORKERS

Intervention acceptability and usefulness as reported by public health workers are reported in tables 2 and 3 .

TABLE 2: NUWBER OF PUBLC HEALTH WORKERS REPORTING THE INTERVEUTION TO BE ACEEPTABLE ( $(N=12)$

\begin{tabular}{lc}
\hline & Yes \\
\hline Role made sufficiently clear? & 12 \\
\hline Should anything else have been included in training? & 0 \\
\hline Workload reasonable? & 11 \\
\hline Worthwhile use of your time? & 10 \\
\hline Public health workers most suitable? & 8 \\
\hline Worked with rugby league in absence of this project? & 5 \\
\hline
\end{tabular}

TABLE 3: NUMBER OF PUBUC HEALTH WORKERS REPORTING THE INTERVENTION COMPONENTS TO BE USEFUL $(N=12)$

\begin{tabular}{lc}
\hline & Useful \\
\hline Training day useful? & 12 \\
\hline Information in kit useful? & 11 \\
\hline Information in kit credible? & 12 \\
\hline Kit user friendly? & 12 \\
\hline Report from lead agency useful? & 12 \\
\hline Contact with police useful? & 7 \\
\hline Police important to project? & 11 \\
\hline Sense of ownership? & 3 \\
\hline
\end{tabular}




\section{Discussion}

In spite of a suggested culture of harmful alcohol consumption among rugby league participants and spectators, ${ }^{11-13}$ non-metropolitan rugby league clubs appear receptive to strategies that increase their responsible service of alcohol. Such a receptiveness provides an opportunity for agencies concerned with reducing alcohol-related harm to work more actively in this setting. The acceptance by rugby league clubs of the intervention strategies provides a basis upon which such agencies may fulfil this opportunity. However, this potential is qualified by not all public health workers reporting such interventions to be part of their role, and only slightly more than half of the clubs reporting the contribution of public health care workers to have been useful.

The receptiveness of rugby league clubs to strategies that aid their responsible service of alcohol supports data from previous research indicating the acceptability of such initiatives to liquor licence holders. ${ }^{716}$ Previous research has also shown that such a receptiveness can be translated into action through a variety of strategies including the provision of sponsorship funds, training and support. ${ }^{9,10,15}$ The findings of this study suggest that the provision of information, resources and support by relevant agencies may hold similar promise for rugby league clubs. Further research is required to determine whether such strategies are capable of increasing the responsible service of alcohol, and importantly, in reducing alcohol-related harm.

The inability to contact $20 \%$ of the clubs (primarily in non-urban areas) imposes some limit on the generalisability of the results. However, despite the bias towards clubs in urban areas being more likely to have been contacted, no differences in the reported acceptability and utility of the intervention was evident between these two groups. The findings therefore appear able to be generalised to all nonmetropolitan rugby league clubs in New South Wales. The extent to which the results may be applicable to other rugby league clubs, other sporting associations or other jurisdictions is unknown. Interpretation of the acceptability and usefulness to public health workers should be undertaken with some caution given the small number of workers involved.

Most interventions to reduce alcohol-related harm focus on single settings such as schools, health care services, workplaces and, more recently licensed premises. ${ }^{10}$ Although intervention in any one setting may be effective in reducing the extent of alcohol-related harm in particular sub-populations, such interventions cannot in isolation provide a solution to the occurrence of alcohol-related harm across a community. Community-based approaches that involve the implementation of interventions across a number of settings are required to achieve such an outcome. ${ }^{16}$ The results of this study suggest the appropriateness of including sporting settings, and in particular rugby league clubs, in such community-based approaches to reducing alcohol-related harm.

Similarly, although previous studies have suggested that various intervention strategies may be effective in increasing the responsible service of alcohol, ${ }^{10.15}$ integrated programs that involve the implementation of a number of strategies, including educative/skill-based approaches such as those used in this study, are more likely to be successful. ${ }^{9}$

The success of public health interventions in community settings is dependent upon public health workers having sufficient professional knowledge, attitudes and skills in inter-sectoral program delivery. In this study, despite the appropriateness of their professional background, one-third of participating workers did not consider that they were suitable agents for the conduct of the intervention. Further, almost half of the clubs reported that contact with public health workers was only slightly or not at all useful, despite reporting that such contact was acceptable in principle. A previous review has identified a lack of skill among public health workers in developing, implementing and evaluating inter-sectoral interventions in sports settings. ${ }^{10}$ The results of this current study provide support for this previous finding, and the subsequent recommendation that programs directed at increasing the capacity of public health workers to undertake inter-sectoral programs be developed.

The dissemination model used in this study of a lead public health agency recruiting locally based agents to implement an intervention program is not an uncommon one. As a large proportion of clubs reported not being contacted by a public health worker $(29 \%)$ or local police (39\%), the results highlight the need for public health agencies to routinely monitor the process of, and compliance with, intervention delivery. ${ }^{17} \mathrm{~A}$ large majority of public health workers indicated they did not feel a sense of ownership of the project. The extent to which such a view was associated with either the non-delivery of intervention components, or the usefulness of public health worker contact as expressed by clubs, is unknown. Further research and evaluation is warranted to identify the factors associated with effective application of this approach to public health intervention delivery.

\section{Acknowledgements}

The authors would like to thank the NSW Drug and Alcohol Directorate for providing the funding for this study, NSW Country Rugby League, NSW Health and participating health workers, the NSW Police Service, participating rugby league clubs, and Ms Sam Fairhall, Project Officer.

\section{References}

1 English DR, Holman CDJ, Milne E, Winter MG, Hulse GK, Codde JP, Bower Cl, Corti B, de Klerk N, Kuniman MW, Kurinczuk JJ, Lewin GF, Ryan GA. The quantification of drug caused morbidity and mortality in Australia. 1995. Canberra, Commonwealth Department of Human Services and Health.

2 National Health Strategy. Enough to make you sick: how income and environment affect health. 1992, Canberra, Australian Institute of Health and Welfare.

3 Saltz R, and Hennessy M. Reducing intoxication in Commercial Establishments: an evaluation of responsible beverage services practices. 1990. Berkeley, CA, Prevention Research Centre.

4 Saltz $R$. The roles of bars and restaurants in preventing alcohol-impaired driving: an evaluation of server intervention. Evaluation and the Health Professions 1997; 10:5-27.

5 Holder $\mathrm{H}$, and Wagenaar $\mathrm{A}$. Mandated server training and reduced 
alcohol-involved traffic crashes: A time series analysis of the Oregon experience. Accident Analysis and Prevention 1994;26:89-97.

6 Stockwell T, Vallis R. Phillips A, et al: Attitudes to Health Promotion in Licensed Premises. Joumal of the Royal Society of Health 1988;6:209-210.

7 Wiggers J, Daly J, Considine R and Hazell T. Prevalence and acceptability of public health initiatives in licensed premises. Australian and New Zealand Journal of Public Health 2000;24:320-322.

8 Licensing Court of NSW. Practice Direction 1/97 - Harm minimisation and responsible service of alcohol. 1998. Sydney, NSW Licensing Court.

9 McKnight AJ, and Streff F. The effect of enforcement upon service of alcohol to intoxicated patrons of bars and restaurants. Accident Analysis and Prevention 1994;26:79-88.

10 National Health and Medical Research Council. Health-promoting Sports, Arts and Racing Settings - New challenges for the health sector. 1996. Australian Government Publishing Service, Canberra.

11 Quarrie KL, Feehan M, Waller AE, Cooke KR, Williams S, and McGee R. The New Zealand Rugby injury and Performance Project: alcohol use patterns within a cohort of rugby players. Addiction 1996;9:1865-1868.
12 Lawson JS, and Evans AR. Prodigious alcohol consumption by Australian rugby league footballers. Drug and Alcohol Review 1992;11:193-195.

13 Tutt D, Bauer L, Edwards C. \& Travers, C. Promoting health in sportunravelling the alcohol ties. Should we start with Rugby League? 1994 Winter School in the Sun (Conference Proceedings). Brisbane, Alcohol and Drug Foundation, Queensland.

14 Oldenburg B, Hardcastle D, French M. How does research contribute to evidence based practice in health promotion? Health Promotion Journal of Australia 1996;6:15-20.

15 Boots K. The Designated Driver Program: An outcome evaluation. Health Promotion Journal of Australia. 1994:4:26-29

16 Hancock L, Sanson-Fisher R, Redman S, Burton R, Burton L, Butler J, Gibberd R, Girgis A. Hensley M, McClintock A, Reid A, Schofield $M$, Tripodi $T$, and Walsh $R$. Community action for cancer prevention: overview of the Cancer Action in Rural Towns (CART) project. Health Promotion International 1996;11:277-289.

17 Hawe P, Degeling D, and Hall J (1990). Evaluating Health Promotion: A health workers guide. Sydney: Maclennan and Petty. 